\title{
Rates of Anomalous Bupropion Prescriptions in Ontario, Canada
}

Leab S. Steele, $M D, P b D^{1-4}$

Erin M. Macdonald, MSc ${ }^{1}$

Tara Gomes, MHSc $\mathrm{c}^{1,4-6}$

Simon Hollands, $M \mathrm{Sc}^{1}$

J. Michael Paterson, MSc ${ }^{1,5,7}$

Mubammad M. Mamdani, PharmD, $\mathrm{MPH}^{1,4-6,8}$

David N. Juurlink MD, $P b D^{1,5,9,10}$

for the Canadian Drug Safety and Effectiveness Research Network

'Institute for Clinical Evaluative Sciences,

Toronto, Ontario, Canada

${ }^{2}$ Family and Community Medicine,

St Michael's Hospital, Toronto, Ontario,

Canada

${ }^{3}$ Department of Family and Community Medicine, University of Toronto, Toronto, Ontario, Canada

${ }^{4}$ Li Ka Shing Knowledge Institute, St Michael's Hospital, Toronto, Ontario, Canada

${ }^{5}$ Institute of Health Policy, Management, and Evaluation, University of Toronto,

Toronto, Ontario, Canada

${ }^{6}$ Leslie Dan Faculty of Pharmacy, University of Toronto, Toronto, Ontario, Canada

${ }^{7}$ Department of Family Medicine, McMaster University, Hamilton, Ontario, Canada

${ }^{8}$ Department of Medicine, St Michael's Hospital, Toronto, Ontario, Canada

${ }^{9}$ Sunnybrook Research Institute, Toronto, Ontario, Canada

${ }^{10}$ Departments of Medicine and Pediatrics University of Toronto, Toronto, Ontario, Canada

Conflicts of interest: During the past 3 years, Mubammad Mamdani bas served on advisory boards and/or received honoraria from Astra Zeneca, Bristol-Myers Squibb, Eli Lilly and Company, Glaxo Smith Kline, Hoffman La Roche, Novartis, Novo Nordisk, and Pfizer. The other authors report no conflicts of interest.

\section{CORRESPONDING AUTHOR}

Leah Steele, MD, PhD

277 Victoria St

Toronto Ontario

1ssteele@gmail.com

\begin{abstract}
PURPOSE Reports of bupropion misuse have increased since it was first reported in 2002. The purpose of this study was to explore trends in bupropion prescribing suggestive of misuse or diversion in Ontario, Canada.
\end{abstract}

METHODS A serial cross-sectional study was conducted of Ontarians aged younger than 65 years who received prescriptions under Ontario's public drug program from April 1, 2000, to March 31, 2013. We determined the number of potentially inappropriate prescriptions in each quarter, defined as early refills dispensed within $50 \%$ of the duration of the preceding prescription, as well as potentially duplicitous prescriptions, defined as similarly early refills originating from a different prescriber and different pharmacy. We replicated these analyses for citalopram and sertraline, antidepressants not known to be prone to abuse.

RESULTS We identified $1,780,802$ prescriptions for bupropion, 3,402,462 for citalopram, and 1,775,285 for sertraline. Rates of early refills for bupropion declined during the study from $4.8 \%$ to $3.1 \%$. In the final quarter, rates of early refills for bupropion were more common than for citalopram (3.1\% vs $2.2 \%$ ) $(P<.001)$ but not for sertraline (3.1\% vs $2.9 \%)(P=.16)$. Potentially duplicitous prescriptions for bupropion increased dramatically, from $<0.05 \%$ of all prescriptions in early 2000 to $0.47 \%$ in early 2013 and by the final quarter were more common than both citalopram $(0.11 \%)$ and sertraline $(0.12 \%)(P<.001)$.

CONCLUSIONS Although no marked differences were seen for early refills of bupropion relative to its comparators, potentially duplicitous prescriptions have increased dramatically in Ontario, suggesting growing misuse of the drug.

Ann Fam Med 2015;13:343-346. doi: 10.1370/afm.1818.

\section{INTRODUCTION}

B upropion is a drug widely prescribed for the treatment of depression and as an adjunct for smoking cessation. It is thought to work by inhibiting neuronal reuptake of dopamine and norepinephrine in the central nervous system. Although the drug was initially thought to confer no risk of addiction, $, 1,2$ reports of bupropion misuse have grown since the phenomenon was first reported in $2002 .{ }^{3}$

People who misuse bupropion sometimes characterize its effects as a cocaine-like high. Bupropion can be misused by ingestion, nasal insufflation, or intravenous injection, although the latter often produces extensive local tissue necrosis. ${ }^{4-9}$ Reports of seizures following bupropion misuse have also been documented. ${ }^{10,11}$ In May 2013, the Chief Coroner for Ontario issued a public health alert following 6 deaths involving bupropion insufflation or injection. ${ }^{12}$

There are no large-scale data on the prevalence of bupropion misuse in the general population. In light of increasing reports of bupropion misuse, we explored prescribing trends of bupropion in Ontario, Canada.

\section{METHODS}

We conducted a population-based study of all publicly funded prescriptions for bupropion, citalopram, and sertraline dispensed between April 1, 2000, 
and March 31, 2013. We used the Ontario Public Drug Benefit Program database, which contains prescription information for patients receiving social assistance at the time their prescriptions were filled. This database has an error rate of less than $1 \% .^{13}$ We excluded prescriptions dispensed to residents in long-term care homes and those aged 65 years and older. In Ontario, persons younger than 65 years are eligible for public drug coverage if they receive social assistance, have high prescription drug costs in relation to their net household income, live in long-term care, are disabled, or receive home care.

\section{Definitions of Anomalous Prescribing}

The analyses included all prescriptions for at least 7 days' duration for citalopram, sertraline, or bupropion. In the primary analysis, we defined early refills as those filled within $50 \%$ of the duration of the preceding prescription for the same drug in the same person. If multiple prescriptions for the same drug at different doses were dispensed on the same day, we did not consider them anomalous if they originated from the same prescriber and pharmacy.

Because early refills are often legitimate, we conducted a sensitivity analysis of prescriptions we deemed more likely to have been filled for purposes of misuse or diversion. These potentially duplicitous prescriptions were refilled prematurely according to the definition above but also originated from a prescriber and a pharmacy different from the immediately preceding prescription. Prescriptions with missing pharmacy or prescriber identification were excluded.

All analyses were conducted at the prescription level. Consequently, if a patient had prescriptions for each of citalopram, bupropion, and sertraline, these were included and analyzed independently.

\section{Statistical Analysis}

We calculated the number and proportion of early refills and potentially duplicitous prescriptions for the study drugs in each quarter. Data were plotted and trends were compared visually. We used generalized estimating equations to compare rates among the 3 drugs in the first and last quarters of the study period while accounting for prescription clustering at the patient level using SAS 9.3 (SAS Institute). All analyses were performed at the Institute for Clinical Evaluative Sciences (http://www.ices.on.ca).

This project was approved by the Research Ethics Board of Sunnybrook Health Sciences Centre, Toronto.

\section{RESULTS}

We studied 1,780,802 prescriptions for bupropion, $3,402,462$ prescriptions for citalopram, and 1,775,285 prescriptions for sertraline during the 13-year study period.

Early refills decreased slightly during the study for all 3 drugs (Figure 1). Early refills for bupropion declined from $4.8 \%$ of all prescriptions in early 2000 to $3.1 \%$ by early 2013 . Similarly, early refills for citalopram decreased from $5.2 \%$ to $2.2 \%$, whereas early refills of sertraline decreased from $4.3 \%$ to $2.9 \%$. In the final quarter, rates of early refills for bupropion were significantly more common than for citalopram $(3.1 \%$ vs $2.2 \% ; P<.001)$ but not for sertraline $(3.1 \%$ vs $2.9 \% ; P=.16)$. In the secondary analysis, there were pronounced differences between bupropion and the 2 comparator drugs (Figure 2). Potentially duplicitous prescriptions for bupropion increased from less than $0.05 \%$ in early 2000 to $0.47 \%$ in early 2013 . In contrast, potentially duplicitous refills for citalopram decreased from $0.25 \%$ to $0.11 \%$, potentially duplicitous refills for sertraline also decreased from $0.15 \%$ to $0.12 \%$. By the final quarter duplicitous prescriptions for bupropion $(0.47 \%)$ were significantly more common than both citalopram $(0.11 \%)$ and sertraline $(0.12 \%)(P<.001)$.

\section{DISCUSSION}

Using population data, we documented a marked increase in the number of potentially duplicitous prescriptions for bupropion after 2008. During the final year of the study, we identified 1,502 potentially duplicitous prescriptions for bupropion. With an average of 32 tablets per prescription, this amount corresponds to approximately 48,000 tablets potentially subject to misuse in our study sample. These findings suggest a troubling phenomenon that bupropion prescriptions are being used recreationally at an increasing rate.

Early refills of all 3 study drugs declined during the study period. Several factors may explain this decline. For the past decade, the adoption of electronic medical records by family physicians has been financially supported in Ontario. ${ }^{14} \mathrm{~W}$ ith increased automation in prescribing, physicians may be better equipped to determine when refills are issued early. Moreover, there has been a widespread transition in Ontario from fee-for-service remuneration to capitation for family physicians. ${ }^{15}$ Physicians working in capitation systems see their patients less frequently than those in fee-forservice practices. ${ }^{16}$ Fewer overall visits to physicians may result in the issuance of fewer prescriptions, some of which might otherwise have been characterized as early refills in our analysis. Finally, in August 2011 the Food and Drug Administration warned of QT interval prolongation associated with citalopram, which correlated with a sharp decrease in the number of pre- 
Figure 1. Early refills for bupropion, sertraline, and citalopram, by quarter.

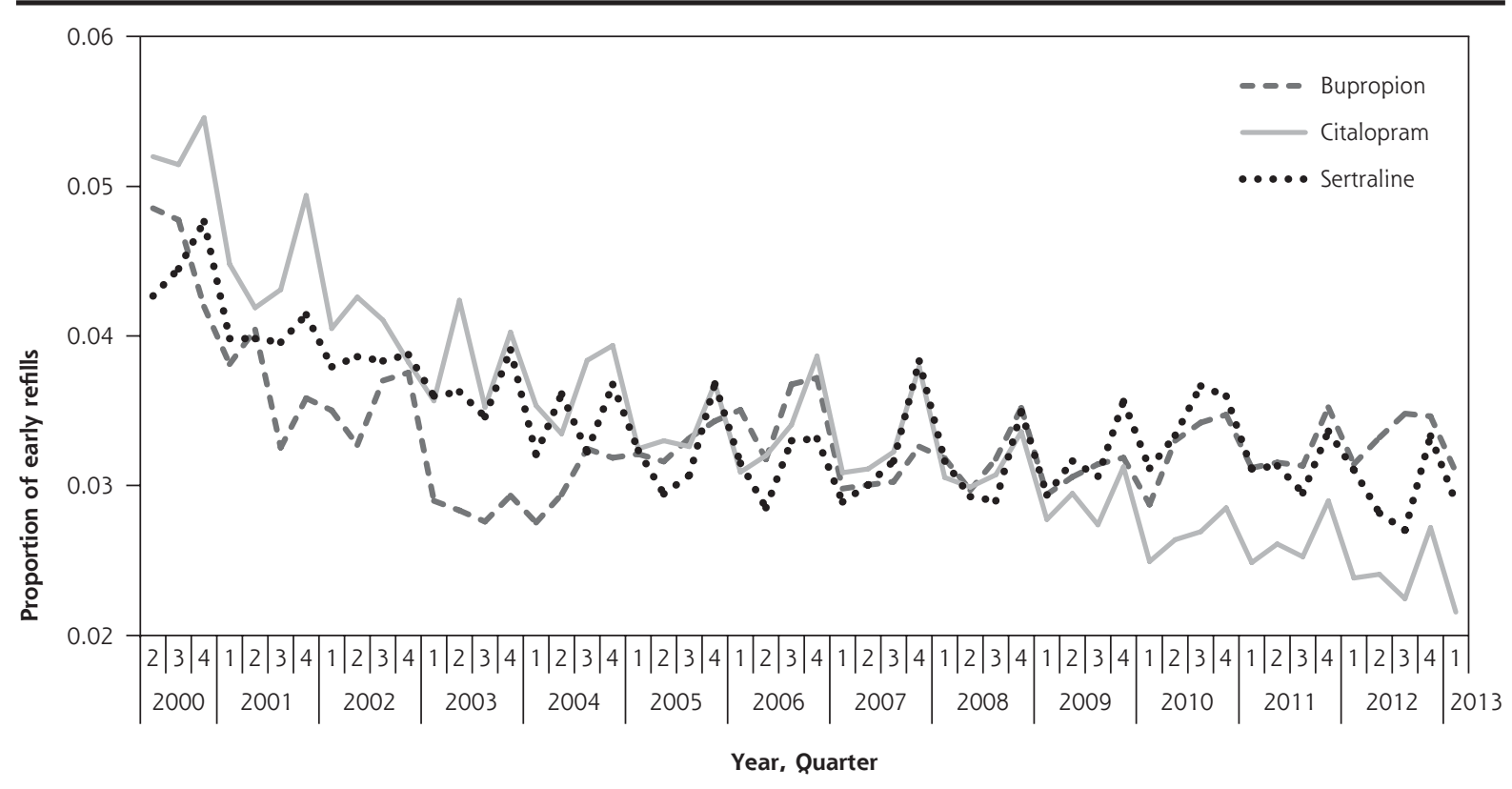

Note: Prescriptions dispensed within 50\% of the days' supply of preceding prescription. Prescriptions for fewer than 7 days of drug use were excluded.

Figure 2. Potentially duplicitous prescriptions for bupropion, sertraline, and citalopram, by quarter.

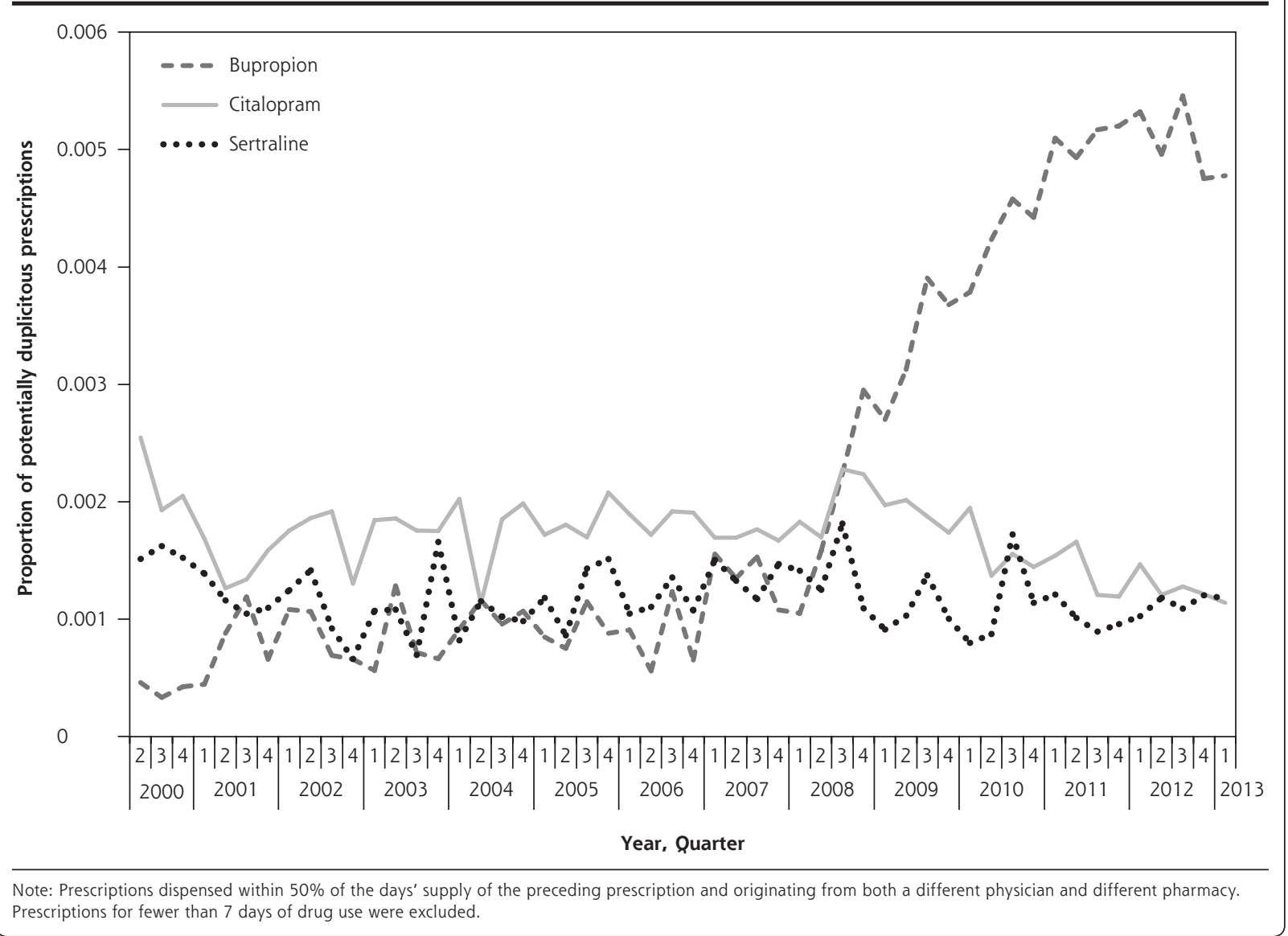


scriptions of citalopram, as well as the number of early refills for that drug. ${ }^{17}$

Some limitations of our study merit emphasis. Defining prescriptions as inappropriate using claims data is an imperfect exercise, and we may have misclassified some appropriate prescriptions. Possible misclassification applies equally, however, to bupropion, sertraline and citalopram. For our secondary analysis, we purposefully used a stricter definition to address this issue. Second, our findings are limited to publicly funded prescriptions among social assistance recipients and may not be generalizable to the entire population. Many individuals with substance use disorders are reliant on the Ontario Drug Benefit Program, however, which accounts for approximately $43 \%$ of all prescription drug expenditures in Ontario..$^{18}$ Consequently, although we identified a large proportion of anomalous bupropion prescriptions in the province, the total number is necessarily higher.

Unlike citalopram and sertraline, bupropion is indicated for the treatment of smoking cessation, and it has been used off-label for the treatment of attention deficit hyperactivity disorder (ADHD). If patients with ADHD require a greater number of legitimate early refills than patients with depression, some of relative increase in anomalous prescribing seen with bupropion may be due to differences in patient characteristics.

We did not find differences in early refills between the 3 drugs; however, we did find a marked rise in the number of potentially duplicitous prescriptions for bupropion. These findings are consistent with growing reports of bupropion misuse. Physicians and pharmacists should be aware of the potential for bupropion misuse, particularly in patients prone to substance use disorders or those who display unusual drug-seeking behaviors. Adding questions about misuse of bupropion to population-based drug use surveys could help determine the extent of the problem.

To read or post commentaries in response to this article, see it online at http://www.annfammed.org/content/13/4/343.

Key words: bupropion; drug abuse; prescription drug diversion

Submitted December 11, 2014; submitted, revised, May 7, 2015; accepted May 22, 2015.

Funding support: This project was supported by research funds from Canadian Drug Safety and Effectiveness Research Network (CDSERN) and by the Institute for Clinical Evaluative Sciences (ICES), which is funded by a grant from the Ontario Ministry of Health and Long-Term Care (MOHLTC).

Disclaimer: The sponsors had no role in the design and conduct of the study; in the collection, analysis, and interpretation of the data; or in the preparation, review, or approval of the manuscript. The opinions, results and conclusions reported herein are those of the authors and are independent from the funding sources. No endorsement by ICES or the Ontario MOHLTC is intended or should be inferred.

Previous presentation: This research was presented at the National American Academy of Clinical Toxicology Conference; October 17-21, 2014; New Orleans, Louisiana.

Acknowledgments: We thank Brogan Inc, Ottawa, for use of their Drug Product and Therapeutic Class Database.

\section{REFERENCES}

1. Griffith JD, Carranza J, Griffith C, Miller LL. Bupropion: clinical assay for amphetamine-like abuse potential. J Clin Psychiatry. 1983;44(5 Pt 2):206-208.

2. Miller L, Griffith J. A comparison of bupropion, dextroamphetamine, and placebo in mixed-substance abusers. Psychopharmacology (Berl). 1983;80(3):199-205.

3. McCormick J. Recreational bupropion abuse in a teenager. Br J Clin Pharmacol. 2002;53(2):214.

4. Phillips D. Wellbutrin: misuse and abuse by incarcerated individuals. J Addict Nurs. 2012;23(1):65-69.

5. Hilliard WT, Barloon L, Farley P, Penn JV, Koranek A. Bupropion diversion and misuse in the correctional facility. J Correct Health Care. 2013;19(3):211-217.

6. Yoon G, Westermeyer J. Intranasal bupropion abuse: case report. Am J Addict. 2013;22(2):180.

7. Reeves RR, Ladner ME. Additional evidence of the abuse potential of bupropion. J Clin Psychopharmacol. 2013;33(4):584-585.

8. Khurshid KA, Decker DH. Bupropion insufflation in a teenager. J Child Adolesc Psychopharmacol. 2004;14(1):157-158.

9. Baribeau D, Araki KF. Intravenous bupropion: a previously undocumented method of abuse of a commonly prescribed antidepressant agent. J Addict Med. 2013;7(3):216-217.

10. Kim D, Steinhart B. Seizures induced by recreational abuse of bupropion tablets via nasal insufflation. CJEM. 2010;12(2):158-161.

11. Hill S, Sikand H, Lee J. A case report of seizure induced by bupropion nasal insufflation. Prim Care Companion J Clin Psychiatry. 2007; 9(1):67-69.

12. Interim Chief Coroner's Alert to Ontario Physicians and Pharmacists. Office of the Chief Coroner [letter]. May 7, 2013. http://www.cfpc. ca/uploadedFiles/Publications/_PDFs/130507\%20Alert\%20to\%20 Ontario\%20Physicians\%20and\%20Pharmacists\%20re\%20Bupropion.pdf. Accessed Mar 31, 2014.

13. Goel V, Williams J, Anderson G, Blackstein-Hirsch P, Fooks C, Naylor D. Patterns of Health Care in Ontario. 2nd Edition ed. Ottawa: Canadian Medical Association; 1996.

14. Biro SC, Barber DT, Kotecha JA. Trends in the use of electronic medical records. Can Fam Physician. 2012;58(1):e21.

15. Glazier RH, Zagorski BM, Rayner J. Comparison of primary care models in Ontario by demographics, case mix and emergency department use, 2008/09 to 2009/10, ICES investigative report, Toronto: Institute for Clinical Evaluative Sciences; 2012.

16. Gosden T, Forland F, Kristiansen IS, et al. Impact of payment method on behaviour of primary care physicians: a systematic review. J Health Serv Res Policy. 2001;6(1):44-55.

17. Federal Drug Administration. FDA drug safety communication: Abnormal heart rhythms associated with high doses of Celexa (citalopram hydrobromide). http://www.fda.gov/Drugs/DrugSafety/ ucm269086.htm. Accessed May 4, 2014.

18. Ontario Ministry of Health and Long-Term Care. 2011/12 Report Card - Ontario Drug Benefit Program. 2012. http://www.health. gov.on.ca/en/public/programs/drugs/publications/opdp/docs/odb_ report_11.pdf. Accessed Apr 5, 2014. 